\title{
Febre maculosa no Brasil: estudo da mortalidade para a vigilância epidemiológica
}

\author{
Spotted fever in Brazil: mortality study for epidemiological \\ surveillance
}

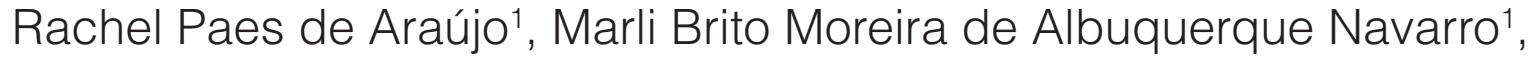 \\ Telma Abdalla de Oliveira Cardoso ${ }^{1}$
}

\begin{abstract}
Resumo
Febre maculosa é uma doença infecciosa caracterizada por febre, com formas leves e atípicas até formas graves, e, quando não tratada adequadamente, pode apresentar taxa de letalidade de $85 \%$. Causada pela Rickettsia rickettsii, é transmitida por meio da saliva de carrapato. No Brasil, o vetor mais importante é o Amblyomma cajennense. Discute-se o número de óbitos por Rickettsia no Brasil, projetando a necessidade de ampliação e disseminação do conhecimento sobre a possibilidade de ocorrência da febre maculosa brasileira (FMB), além de debater sintomas, diagnóstico diferencial e dados epidemiológicos. A metodologia consistiu na identificação dos óbitos por Rickettsia no Brasil entre 2005-2010, utilizando-se o Sistema de Informação sobre Mortalidade (SIM). Foram estudados 75 casos. Observou-se taxa de letalidade de $24,8 \%$ e uma concentração de óbitos nas regiões Sudeste e Sul do país. Os resultados obtidos contribuem para a sensibilização dos profissionais sobre a necessidade de união dos dados clínicos, epidemiológicos e laboratoriais, facilitando o reconhecimento da doença e o tratamento precoce.
\end{abstract}

Palavras-chave: mortalidade; Rickettsia rickettsii; febre maculosa; vigilância epidemiológica.

\begin{abstract}
Spotted Fever is an infectious disease, typically characterized by mild to severe fever, and may reach a $85 \%$ lethality rate if not properly treated. Spotted Fever is caused by Rickettsia rickettsii, and is transmitted through tick's saliva. In Brazil, the most important vector is the Amblyomma cajennense. This study discusses the number of deaths due to rickettsial infection in Brazil, by projecting the needs for expansion and dissemination of knowledge on the possible occurrence of the Brazilian Spotted Fever; we also discuss the symptoms, differential diagnosis and epidemiological data. The methodology consisted in identifying the deaths by rickettsia in Brazil, from 2005 to 2010, using the Mortality Information System. Seventy five cases were studied. There was a concentration of deaths in the southeast and south of the country, and a lethality rate of $24.8 \%$. The results will help in raising awareness among professionals, and will help to understand the importance of combining clinical, epidemiological and laboratory data to facilitate recognition of the disease and early treatment.
\end{abstract}

Keywords: mortality; Rickettsia rickettsia; spotted fever; epidemiological surveillance. 


\section{INTRODUÇÃO}

As rickettsioses são zoonoses de distribuição cosmopolita, de impacto significativo sobre a saúde pública e sobre as atividades econômicas, por atingir a saúde dos animais domésticos, favorecendo a extensão cíclica da cadeia de infecção $0^{1}$. O grupo da febre maculosa, como o tifo exantemático e o tifo do cerrado, causados pela $R$. prowazekii e Orienta tsutugamushi, respectivamente, é o mais importante devido à letalidade expressiva na ausência de tratamento específico ${ }^{2}$.

$\mathrm{O}$ agente etiológico da febre maculosa brasileira (FMB) é uma espiroqueta gram-negativa, de vida intracelular obrigatória, a Rickettsia rickettsii. Ela circula na natureza por meio dos carrapatos, que desempenham um importante papel não só como vetores, mas também como reservatórios, já que podem transmitir a $R$. rickettsii à sua progênie de forma vertical, por transmissão transovariana, e também entre os estádios de sua evolução, por transmissão transestadial e interestadial ${ }^{3}$. Dessa forma, o carrapato permanece infectado durante a vida e por muitas gerações após a infecção primária, garantindo o foco endêmico.

A $R$. rickettsii em animais tem sido detectada em capivaras, gambás, coelhos, equinos, cães e outros animais domésticos e silvestres ${ }^{4}$. É importante destacar o papel destes na cadeia epidemiológica da FMB, pois, além de transportarem os vetores para os domicílios, desempenham papel importante na exaltação da virulência, alimentando os carrapatos ${ }^{5,6}$.

A FMB está normalmente associada às regiões rurais, onde há abundância de animais nos quais o carrapato possa parasitar.

No Brasil, os carrapatos Amblyomma, espécies cajennense, aureolatum. e cooperi ou dubitatum, são seus principais vetores ${ }^{7}$, com destaque para o A. cajennense, conhecido como carrapato-estrela ou rodoleiro, suas ninfas, ou vermelhinhos, e suas larvas, ou "micuins". Apesar disso, qualquer espécie pode ser potencialmente reservatório da $R$. rickettsii, como o Haemaphysalis leporispalustris (carrapato do coelho) e o Rhipicephalus sanguineus (carrapato do cão) ${ }^{8,9}$.

Os carrapatos se infectam ao se alimentarem em animais infectados, transmitindo o patógeno para os animais susceptíveis, mas o homem acaba sendo um hospedeiro acidental ${ }^{3,10}$.

Uma série de fatores favorece a multiplicação do vetor e a expansão da transmissão para além das áreas rurais, atingindo áreas urbanas e periurbanas, tais como: presença de animais hospedeiros; condições ambientais favorecedoras do desenvolvimento e da disseminação dos vetores; meses em que a população de vetores, nas fases parasitárias jovens, está em maior proporção; alterações no ambiente silvestre; influência climática (variações de temperatura, umidade relativa e precipitação pluviométrica); fatores socioeconômicos, culturais e sanitários (proximidade do homem com animais no meio urbano, em especial cavalos e cachorros; banalização do hospedeiro enquanto agente de doença; intensificação do uso da terra e dos sistemas de produção com ocupação desordenada e contínua dos espaços urbanos; colonização de peridomicílios por animais silvestres; hábitos, principalmente, por ecoturismo; controle inadequado do vetor ${ }^{11}$.

A febre maculosa tem sido identificada nos Estados Unidos, Canadá, México, Panamá, Costa Rica, Argentina, Brasil e Colômbia ${ }^{6,12}$.

A vigilância passiva da febre maculosa apresentou uma incidência média anual de 2,2 casos por milhão de pessoas no período de 1997 a $2002^{13}$. No entanto, vem ocorrendo um aumento considerável de casos. Em 2008, o índice chegou a 8,4 casos por milhão de pessoas. Porém a incidência varia por região geográfica ${ }^{14}$.

Dados dos Estados Unidos demonstram maior incidência em crianças ( 5 a 9 anos) e em adultos ( 40 a 64 anos) $)^{12,15}$.

Relatos demonstram que, no passado, os índices de mortalidade alcançavam $87 \%^{13}$. Mesmo com a redução da morbidade e da letalidade pela introdução do cloranfenicol e da tetraciclina, os países da América Latina continuam apresentando altos índices de mortalidade, como a Colômbia $(53 \%)^{13,16}$.

Entre 1997-2009, Del Fiol et al. ${ }^{12}$ identificaram no Brasil 808 casos confirmados de FMB, com taxas de mortalidade entre 20-30\%, ressaltando a tendência na urbanização da doença. Os autores apontaram a notificação, cada vez mais frequente, em São Paulo, Minas Gerais, Santa Catarina, Rio de Janeiro e Espírito Santo.

A febre maculosa é uma patologia multissistêmica considerada grave, com período de incubação de 2-14 dias (média de 7 dias). Seu início súbito e agudo possui sintomas geralmente inespecíficos - tais como febre alta, mialgia, mal-estar generalizado, náuseas e vômitos -, os quais fazem com que a doença seja confundida com leptospirose, dengue, hepatite viral, salmonelose, encefalite malária e pneumonia causada por Mycoplasma pneumonia $e^{5,17}$. O sintoma mais importante é o exantema, que aparece geralmente no terceiro-quinto dia da doença, podendo estar ausente em $9-12 \%$ dos pacientes, dificultando e retardando ainda mais o diagnóstico ${ }^{13}$. Quando essa dificuldade se une ao desconhecimento por parte da população e à desinformação dos serviços de saúde, o número de mortes pode chegar a $80 \%$ dos casos não tratados ${ }^{12}$.

A taxa de mortalidade está relacionada ao diagnóstico precoce e à instituição rápida do tratamento apropriado. Se este for iniciado nos três primeiros dias, essa taxa ficará em torno de $2 \%$ em crianças e $9 \%$ em idosos (acima de 65 anos) ${ }^{12}$.

O diagnóstico laboratorial é feito de duas formas: específico e inespecífico. O primeiro é realizado por meio do isolamento do microrganismo a partir de sangue e tecidos. O segundo detecta anticorpos específicos para $R$. rickettsii, por intermédio da reação de imunofluorescência indireta (RIFI), possuindo sensibilidade 
de $84,6-100 \%$ e especificidade de $99,8-100 \%{ }^{18}$. Em casos mais graves que requerem internação, a coleta de sangue deverá ser feita quando da suspeita clínica. A identificação do DNA da Rickettsia também pode ser feita por PCR. Atualmente, o método mais utilizado no diagnóstico é a sorologia, apesar de os anticorpos IgM e IgG aparecerem somente após 7-10 dias do início da doença ${ }^{19,20}$.

Existem estudos demonstrando a existência de variações intergênicas entre diferentes isolados de $R$. rickettsii associadas à virulência ${ }^{21}$, reforçando a importância não somente da identificação, mas também da caracterização para o entendimento da doença.

Este trabalho objetiva identificar os óbitos causados por FMB no Brasil a fim de discutir suas características epidemiológicas e, assim, contribuir para melhor compreensão da doença pelos profissionais da saúde quanto às ações de vigilância.

\section{MÉTODO}

Estudo descritivo de série histórica, com tempo de seguimento de seis anos (janeiro de 2005 a dezembro de 2010), utilizando dados do Sistema de Informação sobre Mortalidade (SIM) do Ministério da Saúde.

O SIM é o sistema de referência nacional no qual são registradas as causas de morte, baseadas na Classificação Internacional de Doenças e Problemas Relacionados à Saúde (CID) ${ }^{22}$. A CID é a mais recente revisão da "Classificação de Bertillon" de $1893^{23}$, atualmente é um documento oficial da Organização Mundial da Saúde e encontra-se na $10^{a}$ edição.

A população do estudo foi representada pelos óbitos por FMB. Para tanto, foi necessário identificar os óbitos por doenças infecciosas, contidas no capítulo I ("Algumas doenças infecciosas e parasitárias"), especificamente nas categorias A75 a A79 da CID. Como critério de inclusão, buscou-se identificar os óbitos causados pela $R$. rickettsii, inseridos nas categorias A77.0 e A77.9.

Foram excluídos os óbitos por: outra doença infecciosa; doenças cujos agentes etiológicos não foram identificados; doenças não especificadas; onde poderia haver mais de um agente etiológico envolvido.

Os dados foram obtidos no Departamento de Informática do Sistema Único de Saúde (SUS) ${ }^{24}$, disponibilizados no endereço eletrônico <http://sim.saude.gov.br> e acessados no período compreendido entre novembro a dezembro de 2013, utilizando o ambiente operacional do software Oracle 10G.

Como método de análise, foi empregada a distribuição de frequência absoluta, enquanto os dados foram analisados no programa Statistical Package for the Social Science, versão 16.0.

Todos os dados utilizados são de domínio público e preservam a identidade dos indivíduos.

\section{RESULTADOS}

Foi identificado um total de 75 óbitos, distribuídos ao longo de 2005 a 2010, havendo uma variação de incidência por sexo. Observou-se uma predominância entre os homens $(74,7 \%)$ em relação às mulheres $(25,3 \%)$ (Tabela 1$)$, nas faixas etárias compreendidas entre 11 a 20 anos e 21 a 30 anos, apesar de a faixa de 41 a 50 anos também apresentar número expressivo de óbitos (Figura 1). Ainda, com relação à idade dos indivíduos, foi obtida uma média e mediana iguais a 36 e 35 anos, respectivamente, com desvio-padrão de 20,5 anos.

A distribuição dos óbitos causados por $R$. rickettsii por região geográfica mostra uma concentração na região Sudeste (97,3\%) e alguns casos na região Sul (2,7\%). O Estado com maior número de óbitos foi São Paulo (61,3\%), seguido de Minas Gerais (20\%) e Rio de Janeiro (10,7\%) (Figura 2).

O presente estudo mostrou uma redução dos óbitos nos anos de 2007 e 2008. Em 2005, São Paulo apresentou a ocorrência de 16 óbitos e, nos anos subsequentes, um decréscimo de casos, mas que voltou a crescer em 2010 (11 óbitos).

A distribuição temporal dos óbitos pelos meses, ao longo dos anos estudados, é apresentada na Figura 3.

\section{DISCUSSÃO}

A distribuição geográfica dos óbitos registrados é similar aos descritos na literatura sobre a incidência da FMB no Brasil ${ }^{11,12}$.

O Ministério da Saúde ${ }^{20}$ registrou, entre 2001-2008, 601 casos de FMB, com 24,8\% de letalidade, com prevalência na região Sudeste, na qual 267 casos foram registrados em São Paulo, Campinas, Piracicaba e Pedreira. Minas Gerais registrou 104 casos, com destaque para a Região Metropolitana de Belo Horizonte

Tabela 1. Óbitos por Rickettsia rickettsii de acordo com o sexo dos indivíduos, por Estado de ocorrência, de 2005 a 2010

\begin{tabular}{|c|c|c|c|c|c|c|c|c|c|c|c|c|c|}
\hline \multirow{2}{*}{$\begin{array}{l}\text { Unidade da } \\
\text { federação }\end{array}$} & \multicolumn{2}{|c|}{2005} & \multicolumn{2}{|c|}{2006} & \multicolumn{2}{|c|}{2007} & \multicolumn{2}{|c|}{2008} & \multicolumn{2}{|c|}{2009} & \multicolumn{2}{|c|}{2010} & \multirow{2}{*}{ Total } \\
\hline & $\mathbf{M}$ & $\mathbf{H}$ & $\mathbf{M}$ & $\mathrm{H}$ & $\mathbf{M}$ & $\mathbf{H}$ & $\mathbf{M}$ & $\mathbf{H}$ & $\mathbf{M}$ & $\mathbf{H}$ & $\mathbf{M}$ & $\mathbf{H}$ & \\
\hline Paraná & 0 & 0 & 0 & 0 & 0 & 0 & 0 & 0 & 0 & 1 & 1 & 0 & 2 \\
\hline São Paulo & 8 & 8 & 1 & 5 & 1 & 2 & 1 & 3 & 1 & 5 & 2 & 9 & 46 \\
\hline Minas Gerais & 0 & 0 & 0 & 4 & 1 & 2 & 0 & 2 & 1 & 4 & 0 & 1 & 15 \\
\hline Rio de Janeiro & 0 & 0 & 1 & 3 & 1 & 0 & 0 & 2 & 0 & 1 & 0 & 0 & 8 \\
\hline Espírito Santo & 0 & 2 & 0 & 0 & 0 & 0 & 0 & 1 & 0 & 1 & 0 & 0 & 4 \\
\hline Subtotal & 8 & 10 & 2 & 12 & 3 & 4 & 1 & 8 & 2 & 12 & 3 & 10 & 75 \\
\hline Total & \multicolumn{2}{|c|}{18} & \multicolumn{2}{|c|}{14} & \multicolumn{2}{|c|}{7} & \multicolumn{2}{|c|}{9} & \multicolumn{2}{|c|}{14} & \multicolumn{2}{|c|}{13} & 75 \\
\hline
\end{tabular}

$\mathrm{H}=$ homens; $\mathrm{M}=$ mulheres 


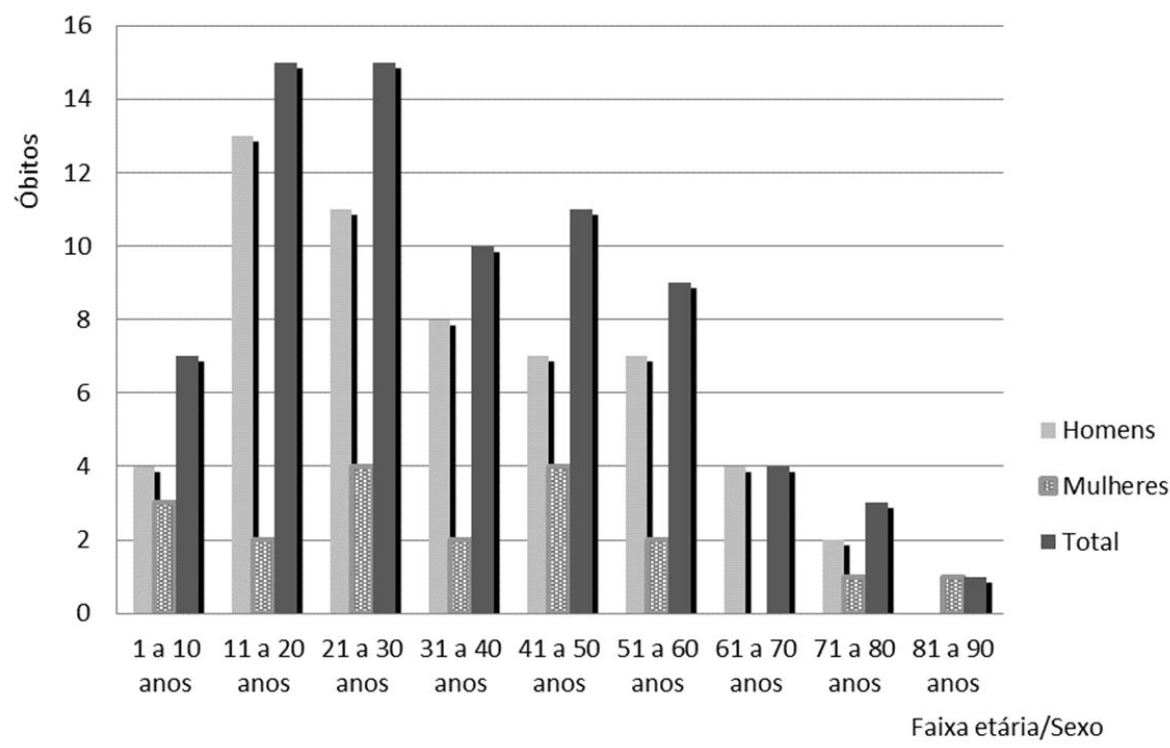

Figura 1. Óbitos de 2005 a 2010 por febre maculosa brasileira (FMB), de acordo com a faixa etária e sexo

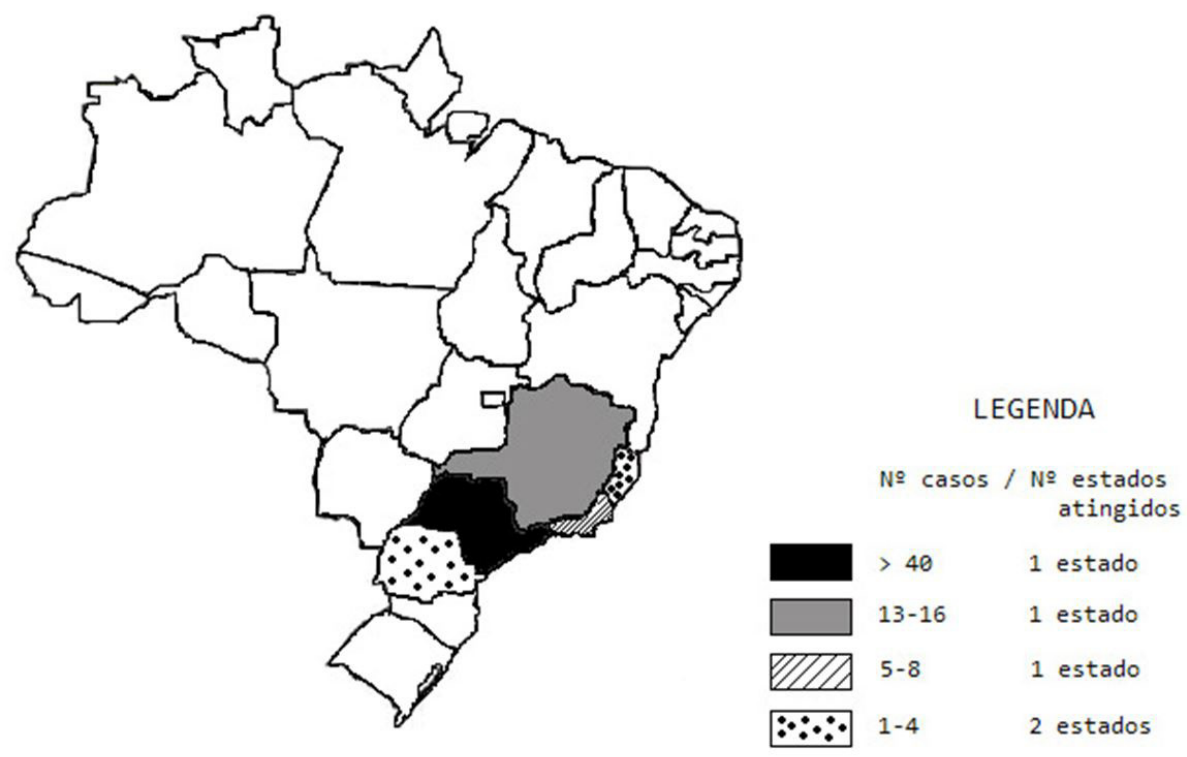

Figura 2. Distribuição no Brasil dos óbitos causados por Rickettsia richettsii no período de 2005 a 2010

e Vales do Rio Doce, Mucuri e Jequitinhonha. A distribuição dos casos restrita a essa região é característica das doenças transmitidas por carrapatos, as quais são normalmente focais, uma vez que os ectoparasitas possuem mobilidade restrita, mas podem ser transportados por animais vertebrados, domésticos, rurais ou silvestres, alastrando a doença $\mathrm{a}^{3,10}$.

Estudos de isolamento da $R$. rickettsii e de detecção de anticorpos por RIFI nas áreas endêmicas de FMB confirmaram a região Sudeste como a de maior número de casos no período de 1985-2012, com taxas de letalidade entre 30-40\% ${ }^{25}$.

O histórico da doença no país pode explicar o maior número de óbitos em São Paulo. Os primeiros relatos da doença ocorreram em 1929 na capital paulista, em áreas onde havia processo de expansão urbana para formação dos bairros atuais de Sumaré, Perdizes e Pinheiros, e nos municípios pertencentes à Região Metropolitana de São Paulo ${ }^{26}$. O primeiro relato de FMB no Rio de Janeiro foi descrito em 1941 em Itaboraí ${ }^{10}$.

A partir de 1948, com a introdução do cloranfenicol e, subsequentemente, da tetraciclina, foi possível verificar uma drástica redução da morbidade e da letalidade nos pacientes tratados precocemente ${ }^{10}$.

Novos casos surgiram na Região Metropolitana de São Paulo somente a partir de 1970. Desde 1985, a doença passou a ser endêmica principalmente em Pedreira e Jaguariúna, na região 


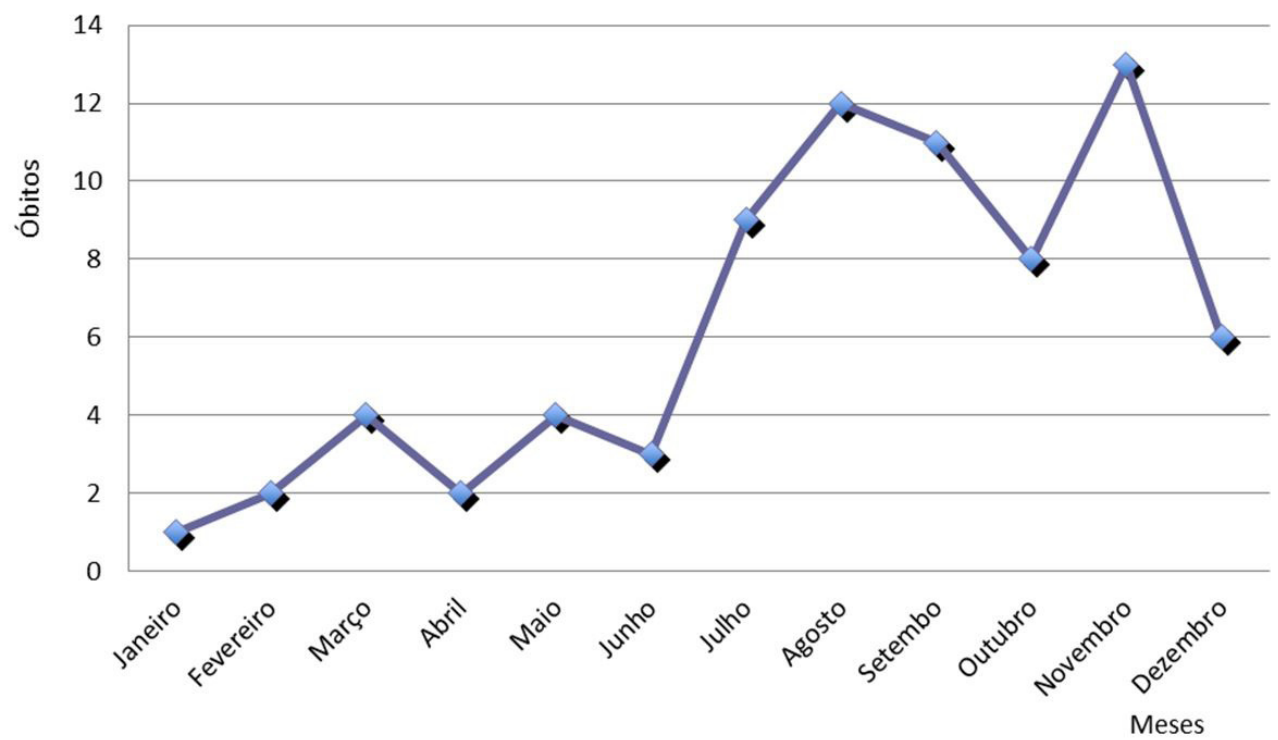

Figura 3. Distribuição dos óbitos por febre maculosa brasileira (FMB) ao longo dos meses, de 2005 a 2010

de Campinas. Nessa mesma época, a doença foi registrada em Minas Gerais, no Vale do Jequitinhonha. O aumento na identificação de casos em São Paulo e Minas Gerais está relacionado à implantação de um programa ativo específico de vigilância epidemiológica pelas Secretarias Estaduais de Saúde, com adoção da notificação compulsória ${ }^{1}$. Por isso, Minas Gerais se destaca com 20\% dos óbitos. Vale observar que esse Estado apresentou em 2009 uma elevação dos óbitos (cinco) e logo no ano seguinte uma redução (um).

A FMB só passou a ser uma doença de notificação compulsória em 2001 pela Portaria nº 1943. Desde então, o número de casos notificados tem aumentado nos Estados do Espírito Santo, Rio de Janeiro e Paraná ${ }^{12}$.

Analisando o ano de 2010, e não contabilizando os 11 óbitos de São Paulo, os demais Estados apresentaram poucos casos. Minas Gerais e Paraná registraram um caso cada, enquanto os demais Estados (Rio de Janeiro e Espírito Santo) não possuíram óbitos.

Os dados apresentados mostram que a FMB ainda é uma doença de impacto em saúde pública, principalmente nos Estados onde há casos fatais. Dados do Instituto Adolfo Lutz demonstram que, apesar de haver uma tendência de queda no número de diagnósticos em São Paulo, sugerindo menor número de casos suspeitos, o número de casos positivos, incluindo óbitos, permanece significativo ${ }^{27}$.

A FMB é esporádica e pontual. Não é frequente a ocorrência de surtos de rápida progressão, uma vez que os carrapatos são ectoparasitas eventuais do homem e geralmente se alimentam de sangue apenas uma vez a cada ciclo ${ }^{28}$.

O período de maior ocorrência de óbitos encontrado neste estudo corresponde à primavera e início do verão, período de maior circulação das fases de larva e ninfa dos carrapatos ${ }^{4,7}$, a despeito de terem sido identificados casos isolados ao longo dos anos estudados. A Tabela 1 mostra que São Paulo, Minas Gerais e Espírito Santo apresentam óbitos de forma reincidente. Uma das justificativas para isso é o ciclo do Amblyomma cajennense. Esse carrapato, responsável pela manutenção da $R$. rickettsii na natureza, necessita de aproximadamente um ano para completar seu ciclo. Porém a incidência da doença está relacionada a fatores como: estágio evolutivo do carrapato, sua distribuição e ecologia; densidade populacional das espécies infectadas que se alimentam no homem contato humano e sua vulnerabilidade à infecção. Dessa forma, a incidência encontrada no estudo, assim como os casos isolados, é resultado da complexa inter-relação, e não somente pela presença dos carrapatos.

Os carrapatos necessitam de condições ambientais, como temperatura, luminosidade e umidade, adequadas para completar o seu ciclo ${ }^{29}$. Observando a Figura 3, observa-se que, nos meses mais quentes, houve um maior número de óbitos, pois ocorre a aceleração da fase de vida livre do vetor, e nos meses mais frios, quando há um retardo no término da fase de vida livre dos carrapatos ${ }^{29}$, os óbitos diminuíram.

As alterações causadas pela atuação do homem no habitat natural dos vetores ou dos hospedeiros da FMB geram desequilíbrios, alterando significativamente a diversidade das interações e proporcionando um aumento na incidência da doença ${ }^{30}$.

Historicamente, a FMB no Brasil foi descrita como sendo uma doença de transmissão em áreas rurais, porém nos últimos anos ocorreram também em áreas periurbanas e urbanas, apesar de as pastagens, matas ciliares e proximidades com rios e lagos serem consideradas ideais para a ocorrência da doença, principalmente com a presença de equinos e de capivaras ${ }^{11}$.

A epidemiologia da doença auxilia na compreensão da prevalência de óbitos no sexo masculino, crianças e adultos 
jovens (faixas entre 11 a 20 e 21 a 30 anos), coincidentes com outros estudos ${ }^{31,32}$. Essa predominância encontra explicação na literatura. A idade adulta está relacionada às atividades ocupacionais nas zonas rurais, de ecoturismo, de exploração de matas ou até mesmo na criação de novas moradias, quando se tem contato com a mata e/ou o foco natural da doença ${ }^{2,31-33}$. Aliado a isso, os homens representam $66,8 \%$ da população rural, e as mulheres, 33,2\% (Censo Agropecuário do Instituto Brasileiro de Geografia e Estatística, 1995-1996) ${ }^{34}$. Assim, justifica-se o maior número de óbitos entre homens.

As crianças, por manterem uma estreita relação com animais domésticos, como o cão, no peridomicílio e maior contato com o solo, tornam-se um grupo vulnerável ${ }^{25,32,33}$.

O número de óbitos observado na faixa acima de 40 anos de idade também foi descrito para a febre das montanhas rochosas ${ }^{35}$. A idade mais avançada (acima de 40 anos) foi apontada como um dos fatores preditores de óbito ${ }^{36}$. Existem ainda relatos sobre a associação de óbitos pela doença à deficiência da glicose-6-fosfato desidrogenase e a patologias concomitantes, como diabetes e alcoolismo ${ }^{12}$.

É uma doença sistêmica, atingindo qualquer órgão ou tecido. Possui uma sintomatologia pouco específica, atrasando o diagnóstico e o estabelecimento de medidas terapêuticas apropriadas, ocasionando alta taxa de mortalidade. Outro fator preditor de óbitos é a ausência ou o retardo no aparecimento de exantema ${ }^{12,32,36}$. Isso demonstra a essencialidade dos cuidados iniciais, requeridos para quaisquer enfermidades de caráter infeccioso, apesar de a doença não ser transmitida de pessoa a pessoa.

Após o surgimento do exantema, a FMB precisa ser diferenciada do sarampo, meningococcemia estafilococcica, infecções por enterovírus, tifo endêmico, febre tifoide, febre amarela, leptospirose, febre purpúrica brasileira, sepse bacteriana com coagulação intravascular disseminada, lues secundária, rubéola, mononucleose infecciosa, entre outras ${ }^{18}$. Com o aumento da incidência de doenças transmitidas por carrapatos, o diagnóstico diferencial deve considerar a doença de Lyme e a erliquiose ${ }^{19}$.

É possível que haja subnotificação de casos em razão do desconhecimento sobre a doença e da dificuldade no diagnóstico clínico, já que pode ser confundida com outras ${ }^{5,37,38}$, evidenciando a importância da vigilância epidemiológica e da necessidade de confirmação diagnóstica dos casos suspeitos. O Ministério da Saúde ${ }^{20}$ define como:

Caso suspeito - indivíduo que apresente febre de moderada a alta, cefaleia, de início súbito, mialgia, história de picada de carrapatos e/ou contato com animais domésticos e/ou silvestres e/ou tenha frequentado área sabidamente de transmissão da Febre Maculosa, nos últimos 15 dias; ou, ainda, indivíduo que apresente febre de início súbito, mialgia, cefaleia, seguida por aparecimento de exantema maculopapular, entre 2 a 5 dias dos sintomas e/ou manifestações hemorrágicas, excluídas outras patologias.

Caso compatível - Indivíduo com clínica sugestiva de FMB que apresente reação sorológica positiva, RIFI com título $\geq 1 / 64$, em amostra única ${ }^{20}$ (p. 186).

O diagnóstico diferencial se faz por meio dos dados clínicos e epidemiológicos associados aos achados laboratoriais. É necessário que os profissionais de saúde reconheçam a sazonalidade da infecção e identifiquem o período de maior incidência de carrapatos para poder orientar a população exposta.

Uma outra razão para a subnotificação é a concentração de casos em zonas rurais, onde podem ocorrer sepultamentos sem a certidão de óbito ${ }^{39}$, causados pela longa distância entre o cartório e a moradia do indivíduo, levando ao sepultamento clandestino ${ }^{40}$.

A legislação brasileira determina a obrigatoriedade da declaração de óbito e do registro do óbito nos cartórios de registro civil para a lavratura da certidão de óbito. Assim, nenhum sepultamento deveria ocorrer sem esse documento, pois este é a base das informações sobre os óbitos para a notificação no SIM.

\section{CONCLUSÃO}

Os óbitos entre 2005-2010 estão concentrados na região Sudeste com $97,3 \%$ dos casos. O Estado com maior número de óbitos é São Paulo, seguido por Minas Gerais, Rio de Janeiro, Espírito Santo e Paraná. Ressalta-se que a falta de sua notificação não reflete a ausência ou a menor intensidade de circulação da $R$. rickettsi na natureza.

A prevalência de óbitos do sexo masculino, crianças e adultos jovens está também associada às degradações ambientais, amplificada por questões econômicas, sociais e biológicas, e expressa pelas vulnerabilidades de ocorrência da doença. Os fatores envolvidos na vulnerabilidade da população diante da infecção por um agente biológico envolvem não só a endemicidade, dose infectante, virulência, modo de transmissão e tratamento, mas também aspectos relacionados à falta de conhecimento, exposição aos patógenos, uso de drogas, fatores genéticos, estado imunológico e nutricional. A vulnerabilidade é uma combinação multifatorial complexa. Esse aspecto possui grande relevância para este estudo, inclusive porque favorece a possibilidade da expansão da doença, sobretudo quando existem variedades de hospedeiros próximos e/ou vinculadas às atividades humanas, como ocorre nas áreas rurais e no ecoturismo. Além disso, as populações das áreas rurais tendem a banalizar o risco relativo ao convívio com o vetor, uma vez que este faz parte de seu cotidiano e por não existirem programas educativos efetivos para demonstrar tais riscos como medida preventiva.

A falta de sensibilização dos profissionais de saúde sobre a possibilidade de ocorrência da FMB pode resultar em elevadas taxas de letalidade, na ausência de diagnóstico e tratamento 
precoce. Essa realidade a coloca dentre as doenças infecciosas negligenciadas. Esse grupo de doenças é resultado da falta de interesse da indústria biotecnológica no desenvolvimento de fármacos, imunobiológicos ou de kits diagnósticos, assim como da insuficiência de políticas públicas, gerando falta de apoio para pesquisa e conhecimento insuficiente sobre o grupo.

Uma das limitações deste estudo está relacionada aos dados sobre a mortalidade coletados do SIM, os quais representam somente uma parcela da população que morre ou que busca hospitalização, que está em estado grave e que tem o seu diagnóstico feito e registrado corretamente. Portanto, os dados não refletem por completo o adoecimento por FMB no Brasil. Os casos brandos passam despercebidos. A falta de testes diagnósticos rápidos faz com que sejam utilizados os diagnósticos mais demorados (isolamento do agente, genoma e detecção do antígeno, por exemplo), quando o paciente pode ir a óbito. Aliado a isso, nos casos fatais, os diagnósticos não foram bem estabelecidos pelos métodos laboratoriais, enquanto que as autópsias foram realizadas em apenas uma baixa proporção de mortes ${ }^{41}$.

Há também as limitações referentes à confiabilidade dos dados, problema relacionado ao preenchimento da declaração de óbito, fonte do SIM. O desconhecimento médico sobre a importância do preenchimento de todos os campos do formulário e sobre o detalhamento da cadeia de eventos patológicos das possíveis causas de morte e a falta de informações suficientes para registrar o óbito estão entre as causas dessa limitação ${ }^{42}$.

É imprescindível para as ações de investigação epidemiológica, vigilância e controle em nível local que os dados tenham qualidade e que os eventos sejam devidamente cobertos. Assim, os médicos devem buscar o nexo epidemiológico para confirmar a doença, no sentido de melhorar as estatísticas de saúde, possibilitando um real diagnóstico da doença na população brasileira.

\section{REFERÊNCIAS}

1. Pinter A, França AC, Souza CE, Sabbo C, Nascimento EMM, Santos FCP, et al. Febre maculosa Brasileira. Bol Epidemiol Paulista. 2011;8(1):3-31.

2. Souza W., coordenador. Doenças negligenciadas. Rio de Janeiro: Academia Brasileira de Ciências; 2010.

3. Burgdorfer W, Brinton LP. Mechanisms of transovarial infection of spotted fever rickettsiae in ticks. Ann N Y Acad Sci. 1975;266(1 Pathobiology):61-72. PMid:829476. http://dx.doi.org/10.1111/j.1749-6632.1975.tb35088.x.

4. Joannitti LHL, Silva NR, D'Auria SRN, Camargo MCGO, Victoria C, Babboni SD, et al. Estimativa de positividade da febre maculosa em cães para a vigilância e o seu monitoramento no município de Botucatu, SP. Vet Zootec. 2014;21(3):451-61.

5. Tiriba AC. Doenças causadas por rickettsias. In: Veronesi R, Focaccia R, organizadores. Tratado de Infectologia. São Paulo: Atheneu; 1999. p. 528-9.

6. Fonseca L, Martins A. Febre maculosa: revisão de literatura-artigo de revisão. Saúde \& Ambiente em Revista 2007;2(1):1-20.

7. Lemos ERS. Rickettsioses. In: Coura JR, organizador. Dinâmica das doenças infecciosas e parasitárias. São Paulo: Guanabara Koogan; 2013. p. 1626-45.

8. Vélez JCQ, Hidalgo M, González JDR. Rickettsiosis, una enfermedad letal emergente y re-emergente en Colombia. Universitas Scientiarum. 2012;17(1):82-99. http://dx.doi.org/10.11144/javeriana.SC17-1.rade.

9. Ogrzewalska M, Saraiva DG, Moraes-Filho J, Martins TF, Costa FB, Pinter A, et al. Epidemiology of Brazilian spotted fever in the Atlantic Forest, state of São Paulo, Brazil. Parasitology. 2012;139(10):1283-300. PMid:22716923. http://dx.doi.org/10.1017/S0031182012000546.

10. McDade JE, Newhouse VF. Natural history of Rickettsia rickettsii. Annu Rev Microbiol. 1986;40(1):287-309. PMid:3096192. http://dx.doi.org/10.1146/ annurev.mi.40.100186.001443.

11. Szabó MPJ, Pinter A, Labruna MB. Ecology, biology and distribuition of spotted-fever tick vectors in Brazil. Front Cell Infect Microbiol. 2013;3:27. PMid:23875178. http://dx.doi.org/10.3389/fcimb.2013.00027.
12. Del Fiol FS, Junqueira FM, Rocha MCP, Toledo MI, Barberato Fo S. A febre maculosa no Brasil. Rev Panam Salud Publica. 2010;27(6):461-6. PMid:20721447. http://dx.doi.org/10.1590/S1020-49892010000600008.

13. Sexton DJ, Walker DH. Spotted fever group rickettsioses. In: Guerrant RL, Walker DH, Weller PF (eds.). Tropical infectious diseases: principles, pathogens, and practice. Philadelphia: Churchill Livingstone; 2006. p. 539-47.

14. Centers for Disease Control and Prevention. Rocky mountain spotted fever. Atlanta; 2015 [citado em 2015 out 2]. Disponível em: http://www.cdc.gov/ rmsf/stats/

15. Dantas-Torres F. Rocky mountain spotted fever. Lancet Infect Dis. 2007;7(11):724-32. PMid:17961858. http://dx.doi.org/10.1016/S14733099(07)70261-X

16. Hidalgo M, Lizarazo D, Ovalle MV, Parra E, Vera M, Porras A, et al. Brote de rickettsiosis en Los Córdobas, departamento de Córdoba, febrero-marzo 2007. Inf Quinc Epidemiol Nac. 2007;12:371-5.

17. Greca H, Langoni H, Souza LC. Brazilian spotted fever: a reemergent zoonosis. J Venom Anim Toxins Incl Trop Dis. 2008;14(1):3-18. http:// dx.doi.org/10.1590/S1678-91992008000100002.

18. Brouqui P, Bacellar F, Baranton G, Birtles RJ, Bjoërsdorff JA, Blanco JR, et al. Guidelines for the diagnosis of tick-borne bacterial diseases in Europe. Clin Microbiol Infect. 2004;10(12):1108-32. PMid:15606643. http://dx.doi. org/10.1111/j.1469-0691.2004.01019.x.

19. São Paulo. Secretaria de Estado da Saúde. Manual de Vigilância Acarológica. São Paulo: SES; 2002.

20. Brasil. Ministério da Saúde. Secretaria de Vigilância em Saúde Doenças Infecciosas e Parasitárias. Doenças Infecciosas e Parasitárias: guia de bolso. 8. ed. Brasília: MS; 2010.

21. Paddock CD, Denison AM, Lash RR, Liu L, Bollweg BC, Dahlgren FS, et al. Phylogeography of Rickettsia rickettsii genotypes associated with fatal Rocky Mountain spotted fever. Am J Trop Med Hyg. 2014;91(3):589-97. PMid:24957541. http://dx.doi.org/10.4269/ajtmh.14-0146. 
22. Organização Mundial da Saúde. Classificação Estatística Internacional de doenças e problemas relacionados à saúde: CID-10. 3. ed. São Paulo: EDUSP; 1996.

23. Laurenti R. Análise da informação em saúde: 1893-1993, cem anos da Classificação Internacional de Doenças. Rev Saude Publica. 1991;25(6):40717. PMid:1843229. http://dx.doi.org/10.1590/S0034-89101991000600001.

24. Brasil. Ministério da Saúde. Secretaria de Vigilância em Saúde. SIM: Sistema de Informação sobre Mortalidade. Brasília: MS; 2015 [citado em 2015 jan 28]. Disponível em: http://sim.saude.gov.br

25. Angerami RN, Câmara M, Pacola MR, Rezende RC, Duarte RM, Nascimento EM, et al. Features of Brazilian spotted fever in two different endemic areas in Brazil. Ticks Tick Borne Dis. 2012;3(5-6):346-8. PMid:23168052. http:// dx.doi.org/10.1016/j.ttbdis.2012.10.010.

26. Piza JT, Meyer JR, Gomes LS. Typho exanthematico de São Paulo. São Paulo: Sociedade Impressora Paulista; 1932

27. Santos FCP. A Importância do Laboratório de Saúde Pública na Vigilância da Febre Maculosa Brasileira no Estado de São Paulo. BEPA. 2009;6(8):51.

28. Sangioni LA, Horta MC, Vianna MC, Gennari SM, Soares RM, Galvao MA, et al. Rickettsial infection in animals and Brazilian spotted fever endemicity. Emerg Infect Dis. 2005;11(2):265-70. PMid:15752445. http:// dx.doi.org/10.3201/eid1102.040656.

29. Veríssimo CJ. Fatores que afetam a fase de vida livre e carrapatos. In: Veríssimo CJ, organizador. Controle dos carrapatos nas pastagens. Nova Odessa: Instituto de Zootecnia; 2013. p. 2-17.

30. Cutler SJ, Fooks AR, Poel WHM. Public health threat of new, reemerging, and neglected zoonoses in the industrialized world. Emerg Infect Dis. 2010;16(1):1-7. PMid:20031035. http://dx.doi.org/10.3201/eid1601.081467.

31. Monteiro KJL, Rozental T, Lemos ERS. Diagnóstico diferencial entre a febre maculosa brasileira e o dengue no context das doenças febris. Rev Patol Trop. 2014;43(3):241-50.

32. Nasser JT, Lana RC, Silva CM, Lourenço RW, Silva DCC, Donalísio MR. Urbanization of Brazilian spotted fever in a municipality of the southeastern region: epidemiology and spatial distribution. Rev Bras Epidemiol. 2015;18(2):299-312. PMid:26083504. http://dx.doi.org/10.1590/19805497201500020002 .
33. Brasil. Ministério da Saúde. Secretaria de Vigilância em Saúde. Guia de vigilância epidemiológica. 7. ed. Brasília: MS; 2009.

34. Instituto Brasileiro de Geografia e Estatística. Orçamento e gestão. Rio de Janeiro: IBGE; 2015 [citado em 2015 jan 28]. Disponível em: http://www. ibge.gov.br/

35. Buckingham SC, Marshall GS, Schutze GE, Woods CR, Jackson MA, Patterson LER, et al. Clinical and laboratory features, hospital course, and outcome of rocky mountain spotted fever in children. J Pediatr. 2007;150(2):180-4, 184.e1. PMid:17236897. http://dx.doi.org/10.1016/j.jpeds.2006.11.023.

36. Díaz JS, Cataño JC. Fiebre manchada de las montañas rocosas: ni tan manchada ni tan montañosa como pensábamos. Infectio. 2010;14(4):26476. http://dx.doi.org/10.1016/S0123-9392(10)70120-X.

37. Pacheco OE, Giraldo MR, Martínez M, Hidalgo M, Echeverri I, Echevarria $\mathrm{L}$, et al. Estudio de brote febril hemorrágico en el corregimiento de Alto de Mulatos-Distrito Especial Portuario de Turbo, Antioquia, enero de 2008. Inf Quinc Epidemiol Nac. 2008;13:145-56.

38. Melles HHB, Colombo S, Lemos ERS. Isolamento de Rickettsia em cultura de células vero. Rev Soc Bras Med Trop. 1999;32(5):469-73. PMid:10881078. http://dx.doi.org/10.1590/S0037-86821999000500001.

39. Frias PG, Pereira PMH, Andrade CLT, Szwarcwald CL. Sistema de Informações sobre Mortalidade: estudo de caso em municípios com precariedade dos dados. Cad Saude Publica. 2008;24(10):2257-66. PMid:18949228. http:// dx.doi.org/10.1590/S0102-311X2008001000007.

40. Frias PG, Vidal SA, Pereira PMH, Lira PIC, Vanderlei LC. Avaliação da notificação de óbitos infantis ao Sistema de Informações sobre Mortalidade: um estudo de caso. Rev Bras Saude Mater Infant. 2005;5(1):S43-51. http:// dx.doi.org/10.1590/S1519-38292005000500005.

41. Galvão MAM, Dumler S, Mafra CL, Calic SB, Chamone CB, Cesarino Fo G, et al. Fatal spotted fever rickettsiosis, Minas Gerais, Brazil. Emerg Infect Dis. 2003;9(11):1402-5. PMid:14718082. http://dx.doi.org/10.3201/ eid0911.030193.

42. Mendonca FM, Drumond E, Cardoso AMP. Problemas no preenchimento da declaração de óbito: estudo exploratório. Rev Bras Estud Popul. 2010;27(2):285-95. http://dx.doi.org/10.1590/S0102-30982010000200004.

Recebido em: Jun. 22, 2015 Aprovado em: Dez. 10, 2015 